\title{
REPORTS OF OBSERVATORIES
}

David Dunlap Observatory. University of Toronto, Richmond Hill, Ontario.

This report covers the interval from July I94I to November 1943. Despite the inroads of the war on the staff of the observatory, we have managed to keep the large telescope employed on every clear night and good progress has been made in the determination of radial velocities and the observation of globular clusters.

The first list of radial velocities of stars published from the observatory was for 500 stars in and near the Kapteyn regions in areas four by four degrees square. These areas were later extended to six by six degrees and included an additional 374 stars. The results for the latter were published in the fall of 1942 as Publications D.D.O. I, No. I3.

On the completion of this second programme we have undertaken the determination of the velocities of all stars appearing in Schlesinger's Catalogue of Bright Stars north of the equator and of types Ao or later for which no velocities were published in January of 1942 . This list includes 684 stars.

The first two programmes were carried through with a camera giving a dispersion of about 60 $\mathrm{A} / \mathrm{mm}$ at $H \gamma$. In starting the third programme we have increased the dispersion to $30 \mathrm{~A} / \mathrm{mm}$. The latter instrument gives much more consistent results. The longer exposure time which would ordinarily be expected with the increased dispersion has been more than counterbalanced by increased efficiency in the optical system.

The observatory constructed a vacuum tank sufficiently large to have the main mirror aluminized and, in addition, the lenses and prism of the spectrograph have been coated with the low reflecting film of fluoride. These two improvements have more than cut the exposures in half so that spectrograms giving a dispersion of $30 \mathrm{~A} / \mathrm{mm}$ at $H \gamma$ can now be secured in less time than it formerly took to get spectrograms with a dispersion of $60 \mathrm{~A} / \mathrm{mm}$.

About 1000 plates remain to be taken on the present programme. It was decided to obtain four plates for each star. As a rule the probable error of the mean of four plates is of the order of $\frac{1}{2} \mathrm{~km} / \mathrm{sec}$.

Miss R. J. Northcott has completed the observation of the spectroscopic binary HD 93075 and the results are published as Communications D.D.O., No. Io.
The observation of globular clusters is under the direction of Dr. Helen S. Hogg and 427 direct photographs have been added to the already extensive collection of this observatory.

Investigations of numerous globular clusters from these plates have continued. In Messier I3 light curves of three long-period Cepheids and one cluster type have been published as Publications D.D.O. I, No. I I, I942. In Messier 56 periods of $\mathrm{I} .53998$ days and $5 \mathrm{I} \cdot 3$ days have been derived for two of the brightest variables, Nos. I and 6 (Pub. Amer. Astr. Soc. 10, 233, I942). Studies of variables in southern globular clusters from plates taken at the Steward Observatory in I939 have shown a long-period Cepheid with a period of 16 days, and four new variables, in Messier 80 (Pub. D.D.O. I, No. I2, I942). The light curve of a semi-regular variable in Messier 4 has been published from over 600 Harvard and Steward observations (Comm. D.D.O., No. 9, I942). In four globular clusters in Ophiuchus, NGC 6273, 6284, 6287, and 6293, 27 new variables have been found. In NGC 6273, variables I and 2 may be long-period Cepheids. Star counts in this cluster confirm its known ellipticity (Pub. D.D.O. I, No. I4, I943).

The two clusters whose investigation is being pressed at present are Messier 22 and Messier I4. From a blink search of Steward plates, 8 new variables have been added to the I7 already known in M 22. Periods have been derived for most of these and for a number of the old variables whose periods were unknown. Periods have been determined for about two dozen of the 72 variables in Messier I4. It is hoped that plates taken during the past two summers when exposures on this object could be shortened from forty minutes to twelve minutes, because of the aluminized mirrors, will permit the determination of many periods in the congested central region of this cluster.

$$
\text { R. K. Young, Director }
$$

Leander McCormick Observatory. University of Virginia, University, Virginia.

The discussion of the observatory's proper motions of faint stars was interrupted last spring, when, after a number of solutions had been made, it was definitely established that the results are much too sensitive to the method of treatment. In particular, the solutions from the $\mu_{\alpha} \cos \delta$ equations tended seriously to disagree with those 
from the $\mu_{\delta}$ equations. It was realized that the chief difficulty lay in the fact that only twothirds of the sky was represented in the solutions so that a clear-cut separation of the seven unknowns was impossible. Consequently, Miss Emma T. R. Williams undertook the reduction of the proper motions of the stars in the Cape zone to the $\mathrm{FK}_{3}$ system by means of the $\mathrm{I} 660$ General Catalogue stars fainter than magnitude 6.9 , which are contained in the zone. Very fortunately, it was found possible to determine the magnitude errors with satisfactory accuracy. With the motions from this zone combined with the McCormick material, more than four-fifths of the sky is represented and the results become very much less sensitive to the method of treatment. Incidentally, the total number of stars involved in this proper motion discussion is approximately twice the number of stars contained in the General Catalogue. Most important of all is the fact that the results from the right ascension equations agree excellently with the independent results from the declination equations. This is a severe test of the dependability of the $\mathrm{FK}_{3}$ system and the astronomers of the Rechen-Institut are to be congratulated. Thus, the right ascension of the apex as determined from the right ascension equations is at $273^{\circ}$ $\pm I^{\circ} .7$, while its position as determined with less weight from the declination equations is at $27 \mathrm{I}^{\circ} \pm 3^{\circ}$.I. The declination of the apex of $+3 \mathrm{I}^{\circ}$ was found from the declination equations and an exactly similar value from the combined equations. It is hoped to start publication of the results early in $\mathbf{I 9 4 4 .}$

Drs. Reuyl and Holmberg have published their discussion of the 70 Ophiuchi system with its evidence of a "planetary" companion of a mass of o.or $\odot$. Dr. Reuyl has also discussed the variable motion from McCormick photographs of Cincinnati I244. The variability is quite marked in both right ascension and declination and the mass of the dark companion is about $0.03 \odot$. A third "planetary" object is in the 6I Cygni system, and it was found by Strand of the Sproul Observatory.

Dr. Vyssotsky has published his first list of 82 dwarf $M$ stars found spectrophotometrically and a discussion of their motions indicates that their mean kinetic energy is about the same as that of A stars. Since this publication, 65 additional dwarf $M$ stars have been detected on the McCormick spectral plates and a preliminary inspection indicates that their peculiar motions are not very different from those of the first list. The consequent inference, that equipartition of energy obtains among all stars of the main sequence, has been confirmed in an interesting manner by the spectral statistics of the second McCormick general proper motion program together with those of the Bergedorf SpectralDurchmusterung. Thus Drs. Vyssotsky and Williams have found that the distribution of stars of the main sequence perpendicular to the galactic plane is just about what might be expected on the assumption that the stars behave like molecules of an isothermal atmosphere. Stars of the giant branch, on the other hand, have much larger kinetic energies and correspondingly smaller galactic concentration. The conclusion that the main sequence stars represent a different system, dynamically, from the stars of the giant branch is borne out by the observation of Miss Williams that the clustering of faint main sequence stars, $\mathrm{A}$ and $\mathrm{F}$, along the Milky Way is quite different from the clustering of the late type giants.

During the summer, the director was at Mount Wilson Observatory and was given the use of the 6o-inch reflector to obtain the radial velocities from spectra of tenth magnitude A and $\mathrm{K}$ stars for which the proper motions are known from McCormick Observatory photographs.

Dr. P. C. Keenan made several short visits to the observatory to investigate the possibilities of the McCormick spectra in connection with the carbon stars.

Miss Edith M. Janssen, an undergraduate at Vassar College, during the summer was engaged in an examination of McCormick spectral plates for dwsrf $M$ stars. In addition to finding 55 dwarf M's, she found four new S stars, and a new emission-line star which is of rare type according to the opinion of Merrill and Joy.

Since the beginning of the year, Dr. Reuyl has been at Aberdeen Proving Grounds where he is head of the photographic division in the Ballistic Research Laboratory.

S. A. Mitchell, Director 